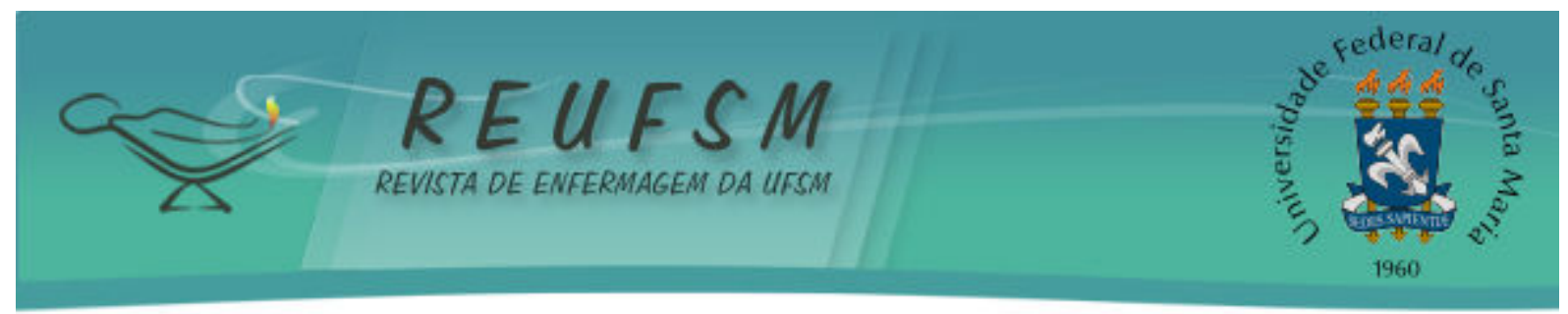

RELATO DE EXPERIÊNCIA

\title{
LABORATÓRIO DE ENFERMAGEM: ESTRATÉGIAS CRIATIVAS DE SIMULAÇÕES COMO PROCEDIMENTO PEDAGÓGICO
}

\author{
NURSING LABORATORY: CREATIVE STRATEGIES USING SIMULATIONS AS AN \\ EDUCATIONAL PROCEDURE
}

\section{LABORATORIO DE ENFERMERÍA: ESTRATEGIAS CREATIVAS DE SIMULACIÓN COMO PROCEDIMIENTO EDUCATIVO}

Ana Elizabeth Figueiredo ${ }^{1}$

\author{
Doi: $10.5902 / 2179769211474$
}

RESUMO: Objetivo: relatar a experiência da utilização de iPads como ferramenta pedagógica e criativa em situações de simulação de aprendizagem no Laboratório de Cuidado Humano ( $\mathrm{LCH}$ ) do curso de Enfermagem. Método: relato de experiência da utilização de tecnologia da informação na simulação realística como estratégia pedagógica para ensino de procedimentos e cuidados de enfermagem. 0 projeto teve início no segundo semestre de 2012, com alunos da graduação de Enfermagem do quinto e sexto nível, o mesmo professor foi responsável por criar e implementar as atividades no LCH. Resultados: houve aumento de $50 \%$ na procura ao LCH para simulação de técnicas como recurso de aprendizado. Os alunos participantes relataram na avaliação institucional das disciplinas uma maior satisfação com o aprendizado de habilidades e raciocínio clínico. Considerações finais: com esta proposta treinamos habilidades que podem resultar em diminuição de eventos adversos para o paciente, garantindo a segurança deste sem prejuízo a aprendizagem dos alunos.

Descritores: Enfermagem; Simulação; Ensino.

ABSTRACT: Objective: to report the experience of using iPads as educational and creative tool in situations of learning simulation in a Human Care Laboratory (HCL) of Nursing. Methods: experience report on the use of information technology in realistic simulation as a pedagogical strategy for teaching procedures and nursing care. The project started in the second semester of 2012, with undergraduate nursing students of the fifth and sixth semesters. The same professor was responsible for creating and implementing the activities in the HCL. Results: there was a 50\% increase in the demand in the $H C L$ for simulation techniques as learning resource. The undergraduate students participating in the simulation reported greater satisfaction with learning abilities and logical reasoning in the subject's institutional evaluation. Final Considerations: with this proposal, we practiced abilities that can result in a decrease of adverse events for patients and enhance their safety without loss for the students' learning process.

Descriptors: Nursing; Simulation; Education.

RESUMEN: Objetivo: relatar la experiencia del uso de iPads como herramienta pedagógica creativa en situaciones de simulación en el Laboratorio de Cuidado Humano (LCH) del curso de Enfermería. Metodos: relato de experiencia del uso de tecnología de la información en simulación realista como estrategia pedagógica para enseñar procedimientos y cuidados de enfermería. El proyecto se inició en el segundo semestre de 2012, con estudiantes de enfermería del quinto y sexto nivel, el mismo profesor fue responsable de la creación e

\footnotetext{
${ }^{1}$ Enfermeira, Pós-Doutor, Pontifícia Universidade Católica Rio Grande do Sul, Porto Alegre, Rio Grande do Sul, Brasil. anaef@pucrs.br
} 


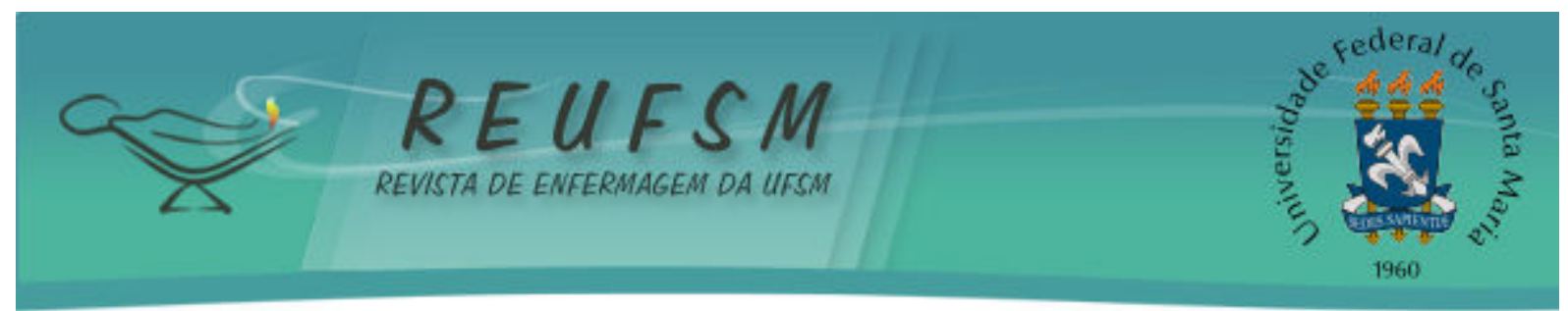

implementación de las actividades en el laboratorio. Resultados: aumentó un 50\% la demanda del LCH para simulación de técnicas como recurso de aprendizaje. Los alumnos participantes relataron en evaluación institucional una mayor satisfacción con el aprendizaje de habilidades y el razonamiento clínico. Consideraciones finales: con esta propuestas entrenamos habilidades que pueden resultar en disminución de eventos adversos para el paciente, garantizando su seguridad sin perjudicar el aprendizaje de los alumnos.

Descriptores: Enfermería; Simulación; Educación.

\section{INTRODUÇÃO}

As escolas de enfermagem devem seguir uma filosofia que possibilite formar profissionais capazes para atender as necessidades de cuidados da população. Espera-se que os profissionais tenham habilidades para o desempenho de técnicas e de raciocínio clínico para a assistência, no entanto, a prática destas habilidades tem campo restrito junto ao paciente. Com o intuito de tornar o aprendizado em laboratório o mais próximo da realidade utiliza-se simulação de casos.

No mundo atual, as tecnologias de informação vêm transformando o mundo levando a modificações profundas em praticamente todas as atividades da sociedade moderna, como o trabalho, a educação, a saúde, a arte e a cultura, dentre outras. ${ }^{1}$

Estas mudanças são vistas entre estudantes de todas as áreas inclusive a da saúde, sendo assim há uma necessidade dos professores repensarem as estratégias de ensino. $\mathrm{Na}$ área da saúde o ensino de habilidades psicomotoras de maneira que sejam experiências satisfatórias para os alunos, foi sempre um desafio. George e Doto ${ }^{2}$ sugerem cinco passos para o ensino de habilidade clínicas, baseado no ensino de habilidade psicomotoras, que consistem em: 1-motivação, onde o aprendiz deve entender o porquê da necessidade da habilidade e como ela é usada no cuidado; 2-o preceptor deve demonstrar a habilidade como ela deve ser feita sem explicações, oferecendo ao aluno uma visão mental do procedimento a ser aprendido; 3-a técnica deve ser repetida com explicações, facilitando ao aluno entender os detalhes desta; 4-a seguir o aluno deve verbalizar a técnica, sendo questionado pelo professor como ela deve ser feita, o que garante que o aluno entenda e lembre o racional para o procedimento; 5-o estudante deve realizar procedimento com supervisão do professor/preceptor, o qual dará retroalimentação durante a realização do procedimento. Estes cinco passos podem ser utilizados para o ensino de qualquer habilidade técnica. ${ }^{2}$

Com os avanços tecnológicos e as novas abordagens pedagógicas, a simulação tem aberto espaços importantes, nas estratégias de aprendizado. ${ }^{3}$ Simulação é definida como aprendizado que amplifica ou repõe situações de vida real, e permite que o aluno pense por meio de uma situação clínica para tomar uma decisão. ${ }^{4}$

As Tecnologias de Informação e Comunicação servem como mais um instrumento de apoio ao professor que pode explorar seu uso para compor situações de simulação que permitam ao aluno praticar ou vivenciar situações abstratas (ou reais) em treinamento ou que ainda não tenha vivenciado. Estes recursos devem ser utilizados de forma complementar ao professor, e não em sua substituição. ${ }^{1}$ A educação, atualmente, precisa caminhar junto com a informatização, pois são inúmeros os novos recursos disponíveis por essa tecnologia. ${ }^{1}$ Os educadores clínicos não devem ver o conhecimento, habilidades e atitudes como um produto do aprendizado, mas sim um conjunto de atributos que são desenvolvidos durante o processo de aprendizagem. ${ }^{5} \mathrm{Um}$ processo orientado de ensino de práticas integradas com tecnologias deve estar incluído num currículo com potencial de facilitar o desenvolvimento de profissionais da saúde graduados e competentes para 


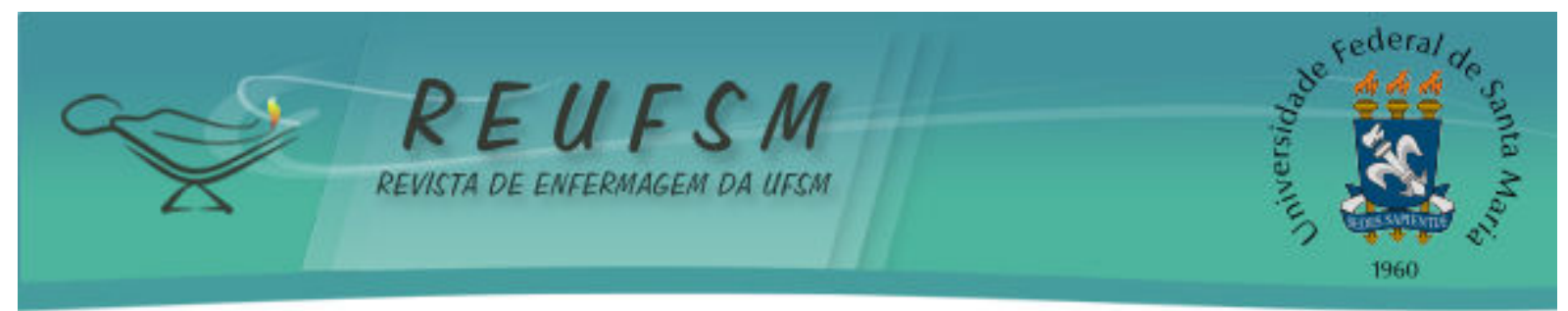

transitar nas complexidades do sistema de saúde, como o gerenciamento dos pacientes além da aplicação de conhecimento e habilidades. ${ }^{5}$

No Brasil, o ensino da enfermagem ainda costuma ocorrer de maneira tradicional, ministrando aulas formais com exposição de conteúdos e pouca participação efetiva dos alunos, onde os laboratórios de enfermagem são visto como laboratório de procedimentos. ${ }^{6}$

Frente à necessidade de mudança na educação clínica, visando uma mudança na formação de profissionais da saúde, onde o ensino seja percebido como mais do que a transmissão de conhecimento e habilidades técnicas, surgiu a demanda de tentar inovar com o uso de iPads como metodologia pedagógica, no ensino de habilidade psicomotoras e pensamento crítico em alunos da graduação de enfermagem. Portanto, o objetivo deste é relatar a experiência da utilização de iPads como ferramenta pedagógica e criativa em situações de simulação no Laboratório de Cuidado Humano do curso de Enfermagem.

\section{METODOLOGIA}

O presente relato descreve a experiência dos iPads nas disciplinas do quinto e sexto nível, Enfermagem Clínica na Saúde do Adulto e Idoso e Enfermagem na Urgência e Emergência, do curso de graduação em Enfermagem da Faculdade de Enfermagem, Nutrição e Fisioterapia, no âmbito do Projeto LabTEAR, em seu subprojeto LabsMóveis, da Pontifícia Universidade Católica do Rio Grande do Sul. A estratégia de ensino e aprendizagem implementada foi a simulação realística que proporciona experiências reais de forma fictícia e segura, tendo como ferramenta os dispositivos móveis. 0 projeto teve início no segundo semestre de 2012, sendo que a criação e implementação das técnicas e situações de simulação foi do professor responsável pelas atividades no Laboratório de Cuidado Humano (LCH).

\section{RESULTADOS E DISCUSSÃO}

Foram empregadas diversas técnicas de simulação como modelo pedagógico em diversos cenários e como manequins, reproduzindo aspectos da realidade de maneira interativa para o grupo, dinamizando o processo de ensino e aprendizagem.

\section{Ensino de habilidades}

Baseado no conceito dos cinco passos para o ensino de habilidades, foi empregado o desenvolvimento de vídeos educativos de determinadas técnicas de enfermagem, tais como: sondagem vesical de demora e alívio, sondagem nasogástrica e nasoentérica e aspiração de vias aéreas. Estes vídeos oferecem ao aluno uma visão geral da técnica a ser aprendida. Os vídeos foram apresentados em sala de aula com as devidas explicações e depois disponibilizados no LCH para que o aluno utilizasse como tutorial para a realização das técnicas desenvolvendo suas habilidades. Notamos que com a disponibilização destes vídeos houve uma maior procura pelo LCH. O aluno busca o LCH para praticar e aperfeiçoar as habilidades necessárias para a prática da enfermagem. Os vídeos servem como reforço da aprendizagem e modelo a ser seguido, reforçando o aprendizado da técnica. A disponibilização dos vídeos educativos aumentou, em mais de 50\%, a procura do LCH para a prática destas habilidades, como forma de tutorial na autoaprendizagem em procedimentos técnicos, até o momento mais de 150 alunos vivenciaram a simulação no $\mathrm{LCH}$. Melo e Damasceno referem que o professor pode explorar o uso do computador para várias situações que permitam a simulação, facilitando o entendimento de realidades abstratas ou reais nas quais o aluno ainda não está preparado para vivenciar. ${ }^{7}$ 


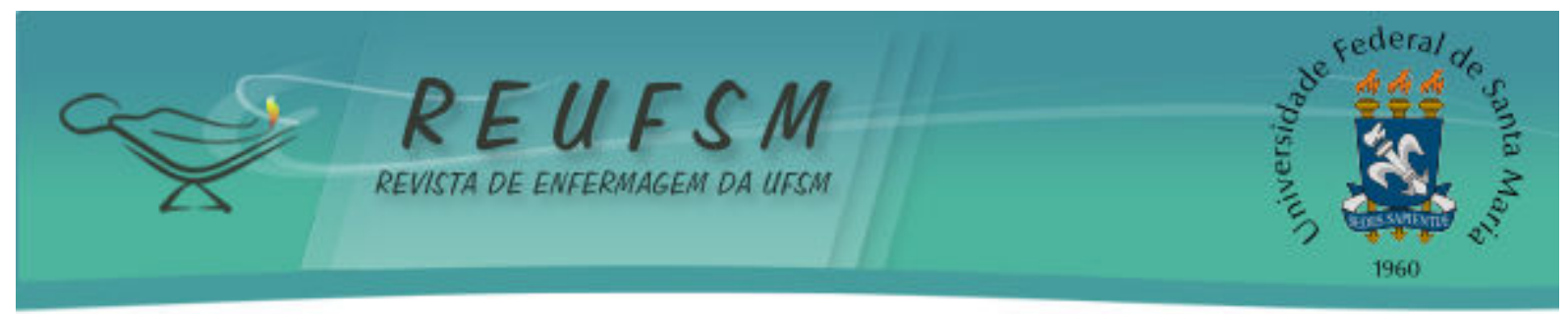

\section{Reflexão crítica}

No intuito de ampliar a discussão de conteúdos sob vários pontos de vista e colocar o aluno como sujeito ativo do seu processo de aprendizagem, outro procedimento pedagógico utilizado em laboratório foi a reflexão crítica de habilidades e procedimento técnicos, onde os alunos eram estimulados a estudar e preparar uma determinada técnica. Após filmarem o procedimento realizado, deveriam fazer a análise crítica reflexiva sobre os acertos, dificuldades e habilidades dominadas durante o procedimento. Como metodologia de ensino, pode ser uma alternativa interessante, pois permite que o aluno realize a análise, interpretação, crítica, levantamento de hipóteses, busca de suposições, obtenção e organização de dados, comparação e aplicação de fatos a novas situações. ${ }^{8}$

Esta mesma abordagem pedagógica também pode ser utilizada em outras situações que não envolvam técnica, como no caso da passagem de plantão. Esta é uma habilidade requerida no enfermeiro que necessita conhecimento, pensamento crítico, determinação de prioridade e habilidade de comunicação, assistir o vídeo com a passagem de plantão ajuda a enxergar as fragilidades e necessidades de aprendizagem. Durante a apresentação, comentários em relação à autoimagem, ao modo de falar e também quanto aos conteúdos apresentados nos vídeos foram comuns por parte dos alunos.

\section{Uso de aplicativos}

Como é de saber comum, as simulações de casos tem a grande vantagem de preparar os alunos para situações-problema que serão encontradas na realidade assistencial. A terceira metodologia empregada foi o uso de um aplicativo de ausculta (cardíaca, pulmonar e abdominal). O uso deste aplicativo e a inovação de colocar um amplificador na campânula do estetoscópio acoplado ao iPad, possibilitou trazer realismo aos casos clínicos. O LCH foi transformado em unidade hospitalar com seis pacientes com diferentes patologias e necessidades de cuidado, requerendo diferentes competências e habilidades. Neste contexto, foi possível a aplicação do processo de enfermagem. Acompanhado do professor, o aluno examinava um dos manequins com uma determinada patologia simulada. Durante o exame físico o professor avaliava a habilidade da realização do exame e o conhecimento de semiologia e semio-técnica. Durante o exame do tórax e abdome do manequim, o professor, com a ajuda do aplicativo, disponibiliza diferentes sons de ausculta pertinente ao caso. $O$ aluno tem então que identificar e correlacionar os achados com o caso clínico em questão, dando realismo a vivência de laboratório. A aprendizagem por simulação é reconhecida como a mais importante inovação no ensino de profissionais da saúde, por propiciar o aprendizado pela prática do fazer, ensaio e desempenho de papéis fictícios, promovendo o entendimento da técnica ou terapêutica a ser aplicada. ${ }^{9}$

0 LCH pode ser transformado em outras unidades, como por exemplo, uma Emergência, com simulações de acolhimento e recebimento de pacientes, aprimorando as habilidades de entrevista e determinação de prioridades no cuidado. É de conhecimento que algumas situações são geradoras de estresse para o acadêmico, uma delas é a Parada Cardiorrespiratória (PCR). 0 estresse inibe e dificulta o desempenho profissional e a aprendizagem, levando a erros, eventos adversos e frustação no atendimento. Com o intuito de minimizar esta experiência negativa, foram criados vídeos, com diversos tipos de monitorização cardíaca, para que o aluno pudesse vivenciar durante a prática assistida em laboratório situações como parada cardiorrespiratória e a possibilidade de atuar na ressuscitação. A simulação levou a aprendizagem no que se refere ao emocional de como lidar com situações de perda e estresse presentes nestes momentos. Nem sempre a 


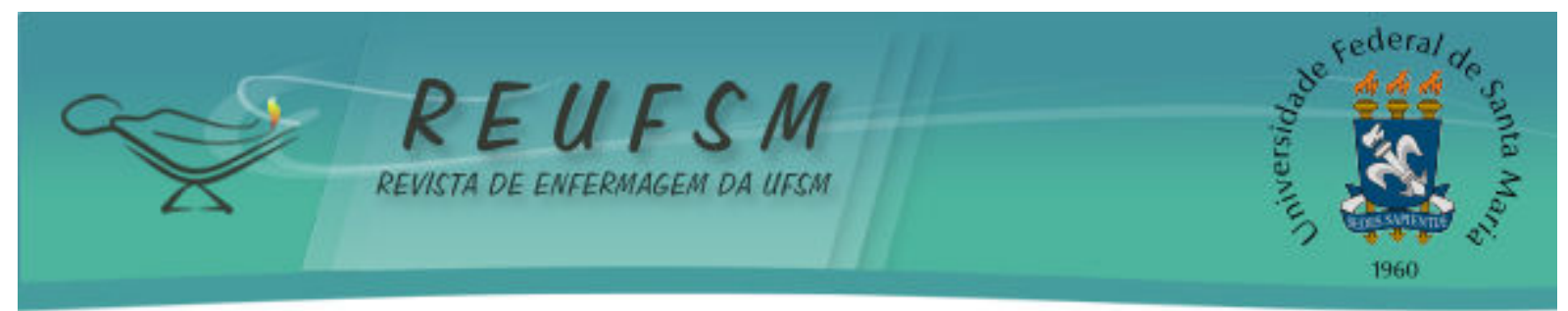

reanimação foi efetiva, seja pela gravidade do paciente ou ineficácia do atendimento prestado. Sabe-se que o manejo de estressores nas profissões da área da saúde são extremamente importantes. ${ }^{10,11}$ Dal Sasso e Souza relatam que o ambiente simulado de aprendizagem assistida por computador, faz com que o aluno seja capaz de ver a realidade simulada e operar sobre ela. Sendo proporcionado ao aluno uma oportunidade nova e estimulante de aprendizagem. ${ }^{11}$

Um estudo australiano relata o modelo STAR (sensibilizar, ação e reflexão), que se refere à necessidade de se sensibilizar com o tema, agir e refletir sobre o que aconteceu, que é apontado pelas autoras como a chave do sucesso. ${ }^{4}$

Teixeira e Félix numa revisão integrativa sobre simulação no ensino da enfermagem, concluíram que a aplicação de estratégias educacionais em laboratório pode ser associada à tecnologia computacional, como apoio ao ensino presencial e referem também que há disposição dos docentes e discentes para formas inovadoras no ensino, particularmente as simulações. ${ }^{12}$

Inovações no ensino da enfermagem utilizando metodologia ativa e participativa mudam o foco da aprendizagem do conteúdo para a aprendizagem significativa, realista com foco no estudante e realismo da assistência. Os alunos participantes relataram na avaliação institucional das disciplinas uma maior satisfação com o aprendizado de habilidades e raciocínio clínico.

Não encontramos na literatura, nenhum relato com a utilização dos iPads, na simulação de situações reais na área da enfermagem.

\section{CONSIDERAÇÕES FINAIS}

Com as inovações propostas a procura ao Laboratório de Cuidado Humano dobrou no primeiro semestre de implementação, o que nos leva a concluir que o uso de técnicas de simulação além de melhorarem as habilidades e competências técnicas dos alunos, tem papel importante no que tange ao aspecto ético-legal e de segurança do paciente. Acredita-se que haverá diminuição de eventos adversos, portanto, o risco do paciente diante da inexperiência do estudante.

\section{REFERÊNCIAS}

1. Fonseca LMM, Góes FSN, Ferecini GM, Leite AM, Scochi CGS. Inovação tecnológica no ensino da semiotécnica e semiologia em enfermagem neonatal: do desenvolvimento à utilização de um software educacional. Texto \& Contexto Enferm. 2009;18(3):549-58.

2. George J, Doto FX. A simple five-step method for teaching clinical skills. Fam Med. 2001;33(8):577-8

3. Azzopardi T, Johnson A, Phillips K, Dickson C, Hengstberger-Sims C, Goldsmith M, et al. Simulation as a learning strategy: supporting undergraduate nursing students with disabilities. J Clin Nurs. 2014 Feb;23(3-4):402-9. doi: 10.1111/jocn.12049. [Epub 2013 Mar 29]

4. McAllister M, Levett-Jones $\mathrm{T}$, Downer $\mathrm{T}$, Harrison $\mathrm{P}$, Harvey $\mathrm{T}$, Reid-Searl $\mathrm{K}$, et al. Snapshots of simulation: creative strategies used by Australian educators to enhance simulation learning experiences for nursing students. Nurse Educ Pract. 2013 Nov; 13(6):567-72. doi: 10.1016/j.nepr.2013.04.010. [Epub 2013 May 23].

5. Rowe M, Frantz J, Bozalek V. Beyond knowledge and skills: the use of a Delphi study to develop a technology-mediated teaching strategy. BMC Medical Education. 2013 [acesso 


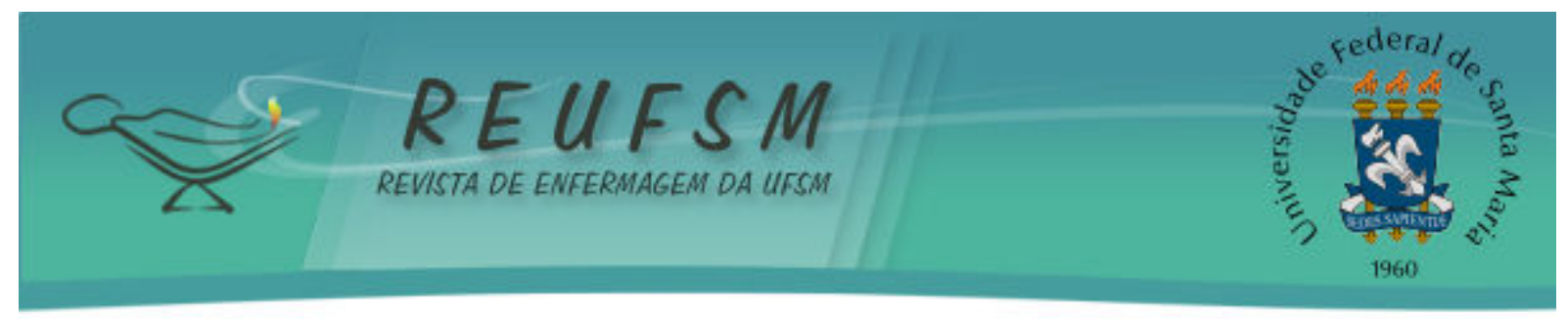

em 2013 jul 10];13:51. Disponível em: http://www.biomedcentral.com/1472-6920/13/51.

6. Felix CCP, Faro ACM, Dias CRF. Percepção de estudantes de enfermagem sobre o Laboratório de Enfermagem como estratégia de ensino. Rev Esc Enferm. 2011;45(1):243-9.

7. Melo FNP, Damasceno MMC. A construção de um software educativo sobre ausculta dos sons respiratórios. Rev Esc Enferm. 2006;40(4)563-9.

8. Anastasiou LGC, Alves LP, organizadoras. Processos de ensinagem na universidade: pressupostos para as estratégias de trabalho em aula. Joinville (SC): UNIVILLE; 2004.

9. Coleman EA. Extending simulation learning experiences to patients with chronic health conditions. JAMA. 2014;311(3):243-4.

10. Pereira MAD, Barbora MA. Teaching strategies for coping with stress- the perceptions of medical students. BCM Medical Education. 2013;13:50.

11. Dal Sasso GTM, Souza MT. A simulação assistida por computador: a convergência no processo de educar-cuidar da enfermagem. Texto \& Contexto Enferm. 2006;15(2):231-9.

12. Teixeira INDO, Felix JVC. Simulação como estratégia de ensino em enfermagem: revisão de literatura. Interface Comun Saúde Educ. 2011;15(39):1173-84.

Data de recebimento: $17 / 11 / 2013$

Data de aceite: $24 / 11 / 2014$

Contato com autor responsável: Ana Elizabeth Figueiredo

Endereço postal: Rua Miracema, 407- Chácara das Pedras - Porto Alegre -91330490

E-mail: anaef@pucrs.br 